\title{
Intrinsic constrains on thermally-assisted memristive switching
}

\author{
Dmitri B. Strukov • R. Stanley Williams
}

Received: 1 December 2010 / Accepted: 22 December 2010 / Published online: 26 January 2011

(c) The Author(s) 2011. This article is published with open access at Springerlink.com

\begin{abstract}
We derived analytical formulas to estimate the effective thermal resistance of a metallic cylinder subjected to Joule heating, surrounded by an insulator and bounded by two constant temperature planes in order to estimate the temperature of the hottest point of the system. These solutions are especially relevant for modeling unipolar resistive switches (or memristive devices), and they provide insight into the performance tradeoffs for a thermally driven reset transition. In particular, our results indicate that a minimum current of $1 \mu \mathrm{A}$ is required to successfully reset a unipolar switch, even under the most favorable conditions.
\end{abstract}

\section{Introduction}

Resistance switching (or memristance $[5,23]$ ) in metal oxide thin-film devices was first observed nearly 50 years ago [9]. Since then, this effect has been reported in a wide range of material systems, including organic films, various transition metal oxides, solid state electrolytes and chalcogenides, which have been extensively reviewed $[7,15,17,26]$. In many cases, the switching mechanism is not fully understood; however, there is often convincing experimental evidence of Joule heating during the switching process. For example, the internal temperature during the reset operation of various unipolar devices has been estimated by the increase in resistance of the high conductance (metallic) state to be over $1000 \mathrm{~K}$ in the case of $\mathrm{NiO}$ films [18]. Significantly less

D.B. Strukov $(\bowtie)$

UC Santa Barbara, Santa Barbara, CA 93106, USA

e-mail: strukov@ece.ucsb.edu

R.S. Williams

Hewlett Packard Laboratories, Palo Alto, CA 94304, USA heating was observed during switching of bipolar $\mathrm{TiO}_{2-x}$ [2] and $\mathrm{SrTiO}_{3}$ [11] devices, which may be due to a lower activation energy for the drift of mobile dopants (vacancies or ions) $[24,25]$ that cause the resistance changes. (Note that elevated temperatures during switching are related to state retention and switching speed characteristics of the device since they provide significant nonlinearity of the ionic drift with applied voltage [25, 28].) Another well-studied class of memristive systems, the phase-change materials, is based on chalcogenide films in which heating by several hundred degrees is required to induce a transition between crystalline and amorphous phases [27].

Heating in memristive devices will inevitably lead to certain performance tradeoffs. For example, the most straightforward application of resistance switching devices is in crossbar digital memories $[8,10,13,16,21]$. For such structures, the temperature increase is caused by Joule heating. This imposes limitations on circuit design and feature size scaling, because of large current densities and thermal crosstalk. Therefore, it is important to study the intrinsic thermal properties of the device structure, which to date has only been done in the context of phase-change memory cells $[4,19]$. In those works, a specific cell structure of phase-change memory was analyzed using numerical simulations. In this paper, we present a more general approach to the problem. We derive analytical formulas for the thermal conductance for a range of device geometries given linear thermal and electron transport model, and discuss the performance (current and power) scaling utilizing appropriate materials properties. For example, one can use the formulas to estimate conducting channel dimensions given particular $I-V$ characteristics for unipolar and certain bipolar memristive devices. Likewise, the results can be directly applied to cells in crossbar digital memories and other circuits based on resistive switching devices [14, 20-22]. 

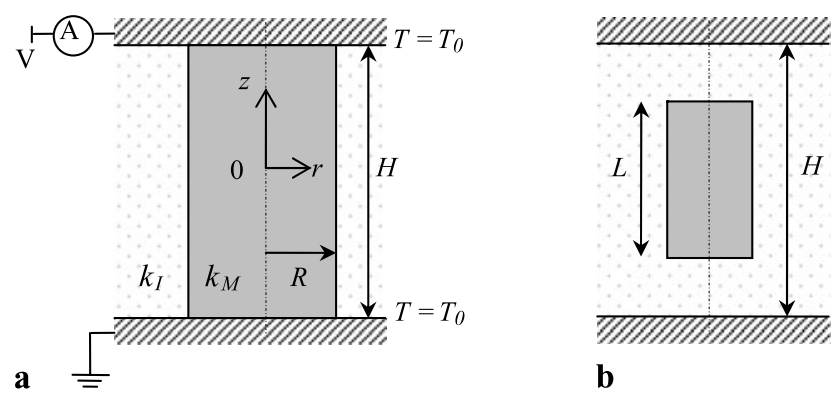

Fig. 1 Schematic cross-section of thin-film device geometries considered in the paper. Here, panel a shows the simplest geometry with a cylinder of radius $R$ and surrounded by an insulator and bounded by constant temperature metallic electrodes. $k_{\mathrm{M}}$ and $k_{\mathrm{I}}$ are the thermal conductivities of the metal rod and insulator, respectively. In panel a the length of the cylinder $L$ is equal to the thickness of the film $H$, while panel $\mathbf{b}$ shows the case when $L<H$. The latter is an approximation to the case where the channel is thinner in the middle of the film, so that the thicker part close to the electrodes is replaced with insulator

\section{Model}

We first consider self-heating in a metallic cylindrical rod surrounded by an insulator and confined by constant temperature planes with geometry and notations presented in Fig. 1a. For the stationary problem, the solution for the temperature distribution in the thin film can be found by solving the Poisson equation

$\Delta T(r, z)=-Q / k_{\mathrm{M}}, \quad r \leq R,-H / 2 \leq z \leq H / 2$

for the metallic cylinder and the sourceless Laplace equation for insulator

$\Delta T(r, z)=0, \quad r \leq R,-H / 2 \leq z \leq H / 2$

using Dirichlet boundary conditions

$T(r,-H / 2)=T(r, H / 2)=T_{0}$.

Here $k_{\mathrm{M}}\left(k_{\mathrm{I}}\right)$ is the thermal conductance of the metallic cylinder (insulating material), assumed to be isotropic, while $q$ is the heat source density generated in the cylindrical metallic rod, which is assumed to be uniform, i.e. $q=I V /\left(L \pi R^{2}\right)$. The Joule heat generated inside the metallic cylinder escapes both radially into the insulator and axially toward the metallic electrodes that are assumed to be large enough to act as thermal sinks. Note that significant heating of electrodes has been observed in some experiments [3]; however, it is rather an artifact of a particular device structure/experiment and should be avoided for practical reasons. For example, electrode heating would exponentially increase diffusion and electromigration of electrode material and therefore degrade endurance.

A general analytical solution for (1)-(3) does not exist. Instead we consider the two limiting cases; first, when heat transfer is mostly via the insulator, i.e. through the sidewalls of the metallic cylinder, and second, when all the heat is transferred through the metallic rod and into the electrodes.

In the first case, the heat transfer along the cylinder is neglected. By slicing the cylinder into thin disks of thickness $d z$, the temperature can be found as the sum of the heating due to each disk. Treating each disk located at point $z$ along the symmetry axis as a point heat source, the temperature at any point $\mathrm{A}$ with coordinates $r_{\mathrm{A}} \geq R,\left|z_{\mathrm{A}}\right| \leq H / 2$ is given by the solution of (2), i.e.

$T_{z}\left(r_{\mathrm{A}}, z_{\mathrm{A}}\right)=q \pi R^{2} d z /\left(4 \pi k_{\mathrm{I}}\right) \times\left(r_{\mathrm{A}}^{2}+\left(z-z_{\mathrm{A}}\right)^{2}\right)^{-1 / 2}$.

The boundary condition, i.e. (3), imposed by constant temperature planes is satisfied using the 'image charge' method. A significant simplification occurs for a thin cylinder with $R \ll L$, so that after integration the temperature at the hottest spot on the surface of the cylinder, i.e. at $z_{\mathrm{A}}=0$ and $r_{\mathrm{A}}=R$ is

$$
\begin{aligned}
T(R, 0) \approx & T_{0}+q R^{2} / 4 k_{\mathrm{I}} \\
& \times(2 \operatorname{ArcSinh}[L /(2 R)]-3 L /(2 H)) .
\end{aligned}
$$

(See details on the derivation of this formula in Appendix.)

In (5) the temperature is given for the more general case shown in Fig. 1b, in which it is assumed that all the heat is dissipated uniformly in a cylinder that is somewhat shorter than the thickness of the device. This geometry is an approximation for a nonuniform radius metallic filament, e.g. thicker close to the electrodes and thinner in the middle, and provides an upper bound for the expected temperature. More specifically, in this case we assume that the gaps between the cylinder and the electrodes have zero electrical resistance, so that all the applied voltage drops across the cylinder of length $L$ while the thermal conductance of the gap is the same as that of the insulator. The upper bound for the temperature increases as the gap between the metallic cylinder and the electrodes gets larger, while for $L=H$ (5) gives a temperature estimate of the geometry shown in Fig. 1a. Given the temperature at the surface of the $\operatorname{rod} T(R, 0)$, the temperature at the center can be approximated by neglecting the boundary conditions and assuming an infinite cylinder, so that (1) is solved for cylindrical coordinates, i.e.

$T_{\mathrm{MAX} \text { THIN }}=T(0,0) \approx T(R, 0)+R^{2} q /\left(4 k_{\mathrm{M}}\right)$.

We now consider the second case of the relatively thick metallic cylinder with $R \gg L$. The solution is obtained by solving the one-dimensional version of (1) along the $z$ direction, resulting in the temperature for the hottest point

$T_{\mathrm{MAX} \text { THICK }}=T(0)=T_{0}+q L^{2} /\left(8 k_{\mathrm{M}}\right)$.

Figure 2a shows the effective thermal resistance $\rho=$ $\left(T-T_{0}\right) /(I V)$ for the hot spot calculated as the parallel 


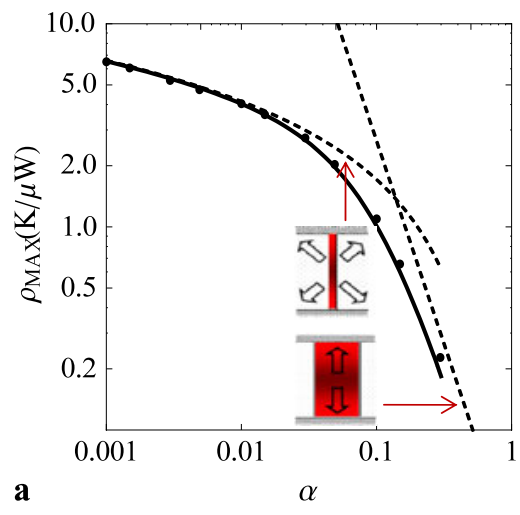

Fig. 2 Effective thermal resistance for the hottest location on the cylinder of a Fig. 1a, and b Fig. $1 \mathrm{~b}$ as a function of $\alpha=R / H$ for $H=50 \mathrm{~nm}, k_{\mathrm{M}}=30 \mathrm{~W} / \mathrm{m} \mathrm{K}$, and $k_{\mathrm{I}}=3 \mathrm{~W} / \mathrm{m} \mathrm{K}$. (Such parameters could be, e.g., representative of titanium oxide thin films with oxygen vacancies conductive channels.) On panel a dashed lines show the con-

combination of the two limiting cases, i.e.

$$
\begin{aligned}
\rho_{\text {MAX }}= & \rho_{\text {MAX THIN }} \times \rho_{\text {MAX THICK }} /\left(\rho_{\text {MAX THIN }}\right. \\
& \left.+\rho_{\text {MAX THICK }}\right),
\end{aligned}
$$

with

$$
\begin{aligned}
\rho_{\text {MAX THICK }}= & L /\left(8 \pi k_{\mathrm{M}} R^{2}\right), \\
\rho_{\text {MAX THIN }}= & 1 /\left(4 \pi k_{\mathrm{M}} L\right)+(2 \operatorname{ArcSinh}[L /(2 R)] \\
& -3 L /(2 H)) /\left(4 k_{\mathrm{I}} L\right),
\end{aligned}
$$

as a function of $\alpha=R / H$ (assuming $L=H$ ) from (6) and (7), for certain practical values of the thickness $H$ and the metal and insulator thermal conductances. The analytical results compare very well with the exact solution obtained via numerical calculations using finite element analysis [6]. Figure $2 \mathrm{~b}$ shows the total thermal resistance for the special case when $L \leq H$ for several values of $L$, which shows that effective thermal resistance is crudely proportional to $H / L$ ratio.

\section{Analysis of the model and implications on scaling}

Equations (8), (9) and Fig. 2 can be used to estimate the radius of the conducting channel in a memristive device given Joule heating information deduced from the experimentally measured resistance increase $[2,18]$ and/or information about the mobile species and reset transition dynamics (i.e. the activation energy for mobile ions and the reset switching time) [25].

This analysis also provides insight into the reset and voltage scaling in unipolar switching devices. For example, if

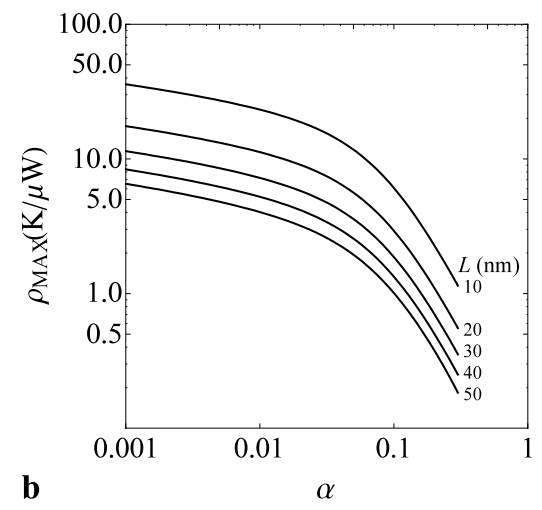

tributions from the radial and axial heat conduction, while the solid line is the total thermal resistance. The black dots show results of numerical simulations, which agree very well with the approximate analytical solution. On panel $\mathbf{b}$, effective total thermal resistance is shown for several values of $L \leq H$

we assume that the electrical current through a cylindrical rod is described by Ohm's law

$I=V \pi R^{2} /\left(\rho_{\mathrm{el}} L\right)$.

Combining this equation with (8) and (9) yields the current (and hence voltage) as a function of the radius of the channel for a given thickness $L$ and temperature increase $\Delta T$ of the metallic cylinder,

$$
\begin{aligned}
I & =\left[\Delta T \pi R^{2} /\left(\rho_{\mathrm{el}} \rho_{\mathrm{MAX}} L\right)\right]^{1 / 2} \\
& \approx 2 \pi k_{\mathrm{I}} \Delta T /\left[\rho_{\mathrm{el}}(\operatorname{ArcSinh}[L /(2 R)]-3 L /(2 H))\right]^{1 / 2},
\end{aligned}
$$

where the approximation is valid for the most interesting case of $R \ll L$.

Figure 3 shows the results of applying (11) to understand reset switching of unipolar memristive devices for several values of $L=H$ (Fig. 1a) and for $L=3 \mathrm{~nm}$ (Fig. 1b). To account for the increase to the bulk electrical resistivity $\rho_{\text {bulk }}$ due to diffusive surface scattering, i.e. when electron mean free path $\lambda$ is smaller or comparable with radius $R$, the following approximation based on Matthiessen rule [12] was used

$\rho_{\mathrm{el}} \approx \rho_{\text {bulk }}[1+\lambda /(2 R)]$.

Each curve for a specific device thickness represents a range of valid cylinder radii, starting with $R=0.3 \mathrm{~nm}$ (always corresponding to the smallest reset current) and the maximum value of $R=0.3 L$ chosen specifically to satisfy considered approximation. One straightforward conclusion from Fig. 3 is that for constant voltage, e.g. a crossbar memory with a pre-determined power supply, there may be no configuration that would allow for heating to a sufficient temperature that enables the reset operation; i.e. the required reset 


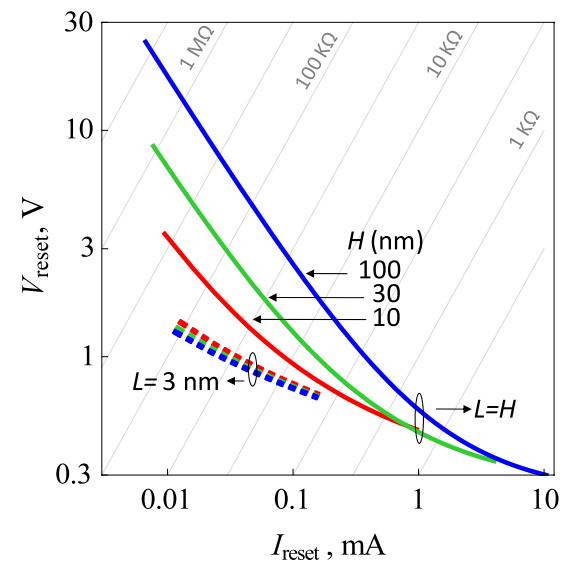

Fig. 3 Reset voltage and current scaling for unipolar resistive switching assuming $k_{\mathrm{M}}=30 \mathrm{~K} / \mathrm{Wm}, k_{\mathrm{I}}=3 \mathrm{~K} / \mathrm{Wm}, \rho_{\text {bulk }}=0.4 \mu \Omega \mathrm{m}$, $\lambda=15 \mathrm{~nm}, \Delta T=700 \mathrm{~K}$ and several values of $H$. Solid and dashed lines show results $L=H$ and $L=3 \mathrm{~nm}$, respectively. Also, for convenience the figure shows constant resistance lines

current may be too large. For example, the smallest value for reset current for considered parameters, even assuming overly optimistic atomic-wide radius of the metallic channel, is around $10 \mu \mathrm{m}$. Note that the much larger effective thermal resistance for the hottest spot for the case when $L \ll H$ results in smaller required power. However, the reset current is about the same as for cases $L=H$ since voltage and current are related by the Ohms law. Also note that though bulk thermal resistivity is used in the calculations it should not affect the most interesting case when $R \ll L$. Thus, for realistic parameters, the reset current needs to be at least in the microampere range, which stresses the driving circuitry and leads to large power consumption in the memory.

\section{Discussion and summary}

We have derived useful analytical equations that allow us to rapidly analyze a wide range of nanoelectronic device configurations in which Joule heating and temperature effects are important. The results agree well with essentially exact numerical simulations, but are accomplished in a small fraction of the time using modest computational resources. We have applied these equations to analyze reset switching for a general class of unipolar memristive devices, and found that the reset current for such devices needs to be at least in the microampere range, which means that the operating power for memories based on such cells will be high. Alternatively, the results show that for nonvolatile memristive device with switching currents below $\sim 1 \mu \mathrm{A}$ nonlinearity in memory phenomena could not be due to Joule heating but rather other phenomena, e.g. exponential ionic drift in electric field.
Acknowledgements We thank J. Borghetti, J.P. Strachan, and J. Yang for useful discussions. Work at UCSB is partially funded by Air Force Office of Scientific Research under Contract No. FA955010-C-0083 and by grant from by NSF grant CCF-1017579.

Open Access This article is distributed under the terms of the Creative Commons Attribution Noncommercial License which permits any noncommercial use, distribution, and reproduction in any medium, provided the original author(s) and source are credited.

\section{Appendix}

To satisfy the condition for constant temperature on the surface of the top electrode, i.e. $T(r, H / 2)=T_{0}$ for a slice of a cylinder positioned at $z=0$ (considered to be a point source), an image sink (heat absorber) is placed at the point $z=H$. This artificial image sink now requires putting another source at $z=-2 H$ to satisfy the boundary condition for the bottom electrode, i.e. $T(r,-H / 2)=T_{0}$. The procedure of installing image heat sources and sinks is carried on indefinitely. Integrating the heat contribution of the entire cylinder with corresponding image sources and sinks yields the temperature

$$
\begin{gathered}
T(r, z)-T_{0}=\frac{Q R^{2}}{4 k_{I}} \int_{-L / 2}^{L / 2}\left(\frac{1}{\sqrt{\left(z^{\prime}-z\right)^{2}+r^{2}}}\right. \\
+\sum_{n=1}^{\infty} \frac{(-1)^{n}}{\sqrt{\left.\left[(-1)^{n}\left(z^{\prime}-n H\right)-z\right)\right]^{2}+r^{2}}} \\
\left.+\sum_{n=1}^{\infty} \frac{(-1)^{n}}{\sqrt{\left.\left[(-1)^{n}\left(z^{\prime}+n H\right)-z\right)\right]^{2}+r^{2}}}\right) d z^{\prime}
\end{gathered}
$$

where the first and the second infinite sums reflects boundary conditions on the surface of the top and the bottom electrodes, respectively.

Since (A.1) is only valid for $r \geq R$, it is clear that the highest temperature will be at the point $z=0$ and $r=R$. Assuming the thin cylinder case, $R \ll L$, (A.1) for the hottest point can be simplified to

$$
\begin{aligned}
T_{\mathrm{MAX}} & (R, 0)-T_{0}=\frac{Q R^{2}}{4 k_{\mathrm{I}}} \int_{-1 / 2}^{1 / 2}\left(\frac{1}{\sqrt{z^{\prime \prime 2}+(R / L)^{2}}}\right. \\
& +\sum_{n=1}^{\infty} \frac{(-1)^{n}}{\sqrt{\left(z^{\prime \prime}-n H / L\right)^{2}+(R / L)^{2}}} \\
& \left.+\sum_{n=1}^{\infty} \frac{(-1)^{n}}{\sqrt{\left(z^{\prime \prime}+n H / L\right)^{2}+(R / L)^{2}}}\right) d z^{\prime \prime} \\
\approx & \frac{Q R^{2}}{4 k_{\mathrm{I}}} \int_{-1 / 2}^{1 / 2}\left(\frac{1}{\sqrt{z^{\prime \prime 2}+(R / L)^{2}}}+\sum_{n=1}^{\infty} \frac{(-1)^{n}}{n H / L-z^{\prime \prime}}\right.
\end{aligned}
$$




$$
\begin{aligned}
& \left.+\sum_{n=1}^{\infty} \frac{(-1)^{n}}{n H / L+z^{\prime \prime}}\right) d z^{\prime \prime} \\
\approx & \frac{Q R^{2}}{4 k_{\mathrm{I}}}(2 \operatorname{ArcSinh}[L / 2 R]-3 L / 2 H) .
\end{aligned}
$$

The final simplification in (A.2) is due to the fact that the infinite sum is

$$
\begin{aligned}
\int_{-1 / 2}^{1 / 2} & \left(\sum_{n=1}^{\infty} \frac{(-1)^{n}}{n H / L-z^{\prime \prime}}+\sum_{n=1}^{\infty} \frac{(-1)^{n}}{n H / L+z^{\prime \prime}}\right) d z^{\prime \prime} \\
= & \frac{1}{2} \int_{-1 / 2}^{1 / 2}\left(\psi\left[1 / 2-z^{\prime \prime} L /(2 H)\right]-\psi\left[1-z^{\prime \prime} L /(2 H)\right]\right. \\
& \left.+\psi\left[1 / 2+z^{\prime \prime} L /(2 H)\right]-\psi\left[1+z^{\prime \prime} L /(2 H)\right]\right) d z^{\prime \prime} \\
= & -2(\ln G[1 / 2-L /(4 H)]-\ln G[1 / 2-L /(4 H)] \\
& +\ln G[1+L /(4 H)]-\ln G[(2 H+L) / 4 H]) \\
\approx & -3 L /(2 H)
\end{aligned}
$$

for $0 \leq H / L \leq 1$, where $\psi$ and $\ln G$ are digamma and logarithmic gamma function, respectively [1].

\section{References}

1. M. Abramowitz, I.A. Stegun, Handbook of Mathematical Functions: with Formulas, Graphs, and Mathematical Tables (Dover, New York, 1965)

2. J. Borghetti, D.B. Strukov, M. Pickett, J. Yang, R.S. Williams, Electrical transport and thermometry of electroformed titanium dioxide memristive switches. J. Appl. Phys. 106, 124504 (2009)

3. S.H. Chang, S.C. Chae, S.B. Lee, C. Liu, T.W. Noh, J.S. Lee, B. Kahng, J.H. Jang, M.Y. Kim, D.-W. Kim, C.U. Jung, Effects of heat dissipation on unipolar resistance switching in $\mathrm{Pt} / \mathrm{NiO} / \mathrm{Pt}$ capacitors. Appl. Phys. Lett. 92, 183507 (2008)

4. Y.C. Chen, C.T. Rettner, S. Raoux, G.W. Burr, S.H. Chen, R.M. Shelby, M. Salinga, W.P. Risk, T.D. Happ, G.M. McClelland, M. Breitwischt, A. Schrott, J.B. Philipps, M.H. Lee, R. Cheek, T. Nirschl, M. Lamorey, C.F. Chen, E. Joseph, S. Zaidi, B. Yee, H.L. Lung, R. Bergmann, C. Lam, Ultra-thin phase-change bridge memory device using GeSb, in Proc. International Electron Devices Meeting, San Francisco, CA, December 2006, art. 4154329 (2006)

5. L.O. Chua, S.M. Kang, Memristive devices and systems. Proc. IEEE 64, 209-223 (1976)

6. COMSOL software. Available online at http://www.comsol.com

7. G. Dearnaley, A.M. Stoneham, D.V. Morgan, Electrical phenomena in amorphous oxide films. Rep. Prog. Phys. 33, 1129-1192 (1970)

8. J.E. Green, J.W. Choi, A. Boukai, Y. Bunimovich, E. JohnstonHalperin, E. DeIonno, Y. Luo, B.A. Sheriff, K. Xu, Y.S. Shin, H.-R. Tseng, J.F. Stoddart, J.R. Heath, A 160-kilobit molecular electronic memory patterned at $10^{11}$ bits per square centimetre. Nature 445, 414-417 (2007)

9. M.T. Hickmott, Low-frequency negative resistance in thin anodic oxide films. J. Appl. Phys. 33, 2669-2682 (1962)
10. S.H. Jo, K.-H. Kim, W. Lu, High-density crossbar arrays based on a Si memristive system. Nano Lett. 9, 870-874 (2009)

11. S.F. Karg, G.I. Meijer, J.G. Bednorz, C.T. Rettner, A.G. Schrott, E.A. Joseph, C.H. Lam, M. Janousch, U. Staub, F. La Mattina, S.F. Alvarado, D. Widmer, R. Stutz, U. Drechsler, D. Caimi, Transition-metal oxide-based resistance change memories. IBM J. Res. Dev. 52(4/5), 481-492 (2008)

12. C. Kittel, Introduction to Solid State Physics, 7th edn. (Wiley, New York, 1995)

13. M.-J. Lee, C.B. Lee, S. Kim, H. Yin, J. Park, S.E. Ahn, B.S. Kang, K.H. Kim, G. Stefanovich, I. Song, J.H. Lee, S.W. Kim, S.J. Chung, Y.H. Kim, C.S. Lee, J.B. Park, I.G. Baek, C.J. Kim, Y. Park, Stack friendly all-oxide 3D RRAM using GaInZnO peripheral TFT realized over glass substrates, in Proc. International Electron Devices Meeting, San Francisco, CA, December 2008, art. 4796620 (2008)

14. K. Likharev, A. Mayr, I. Muckra, Ö. Türel, CrossNets: highperformance neuromorphic architectures for CMOL circuits. Ann. N.Y. Acad. Sci. 1006, 146-163 (2003)

15. K.K. Likharev, CMOL technology: devices, circuits, and architectures. J. Nanoelectron. Optoelectron. 3, 203-230 (2008)

16. T. Mikolajick, M. Salinga, M. Kund, T. Kever, Nonvolatile memory concepts based on resistive switching in inorganic materials. Adv. Mater. 11(4), 235-240 (2009)

17. H. Pagnia, N. Sotnik, Bistable switching in electroformed metalinsulator-metal devices. Phys. Status Solidi A 108(11), 11-65 (1988)

18. U. Russo, D. Ielmini, C. Cagli, A.L. Lacaita, S. Spigat, C. Wiemert, M. Peregot, M. Fanciulli, Conductive-filament switching analysis and self-accelerated thermal dissolution model for reset in NiO-based RRAM, in Proc. International Electron Devices Meeting, Baltimore MD, December 2007, pp. 775-778, art. 4419062 (2007)

19. U. Russo, D. Ielmini, A. Redaelli, A.L. Lacaita, Modeling of programming and read performance in phase-change memories-part I: cell optimization and scaling. IEEE Trans. Electron Devices 55(2), 506-514 (2008)

20. G.S. Snider, R.S. Williams, Nano/CMOS architectures using a field-programmable nanowire interconnect. Nanotechnology $\mathbf{1 8}$, 035204 (2007)

21. D.B. Strukov, K.K. Likharev, Defect-tolerant architectures for nanoelectronic crossbar memories. J. Nanosci. Nanotechnol. 7, 151167 (2007)

22. D.B. Strukov, K.K. Likharev, Reconfigurable hybrid CMOS/ nanodevice circuits for image processing. IEEE Trans. Nanotechnol. 6, 696-710 (2007)

23. D.B. Strukov, G.S. Snider, D.R. Stewart, R.S. Williams, The missing memristor found. Nature 453, 80-83 (2008)

24. D.B. Strukov, J.L. Borghetti, R.S. Williams, Coupled ionic and electronic transport model of thin-film semiconductor memristive behavior. Small 5(9), 1058-1063 (2009)

25. D.B. Strukov, R.S. Williams, Exponential ionic drift: fast switching and low volatility of thin film memristors. Appl. Phys. A 94(3), 515-519 (2009)

26. R. Waser, M. Aono, Nanoionics-based resistive switching memories. Nat. Mater. 6, 833-840 (2007)

27. M. Wuttig, N. Yamada, Phase-change materials for rewriteable data storage. Nat. Mater. 6, 824-832 (2007)

28. V.V. Zhirnov, R.K. Cavin, S. Menzel, E. Linn, S. Schmelzer, D. Brauhaus, C. Schindler, R. Waser, Memory devices: energyspace-time tradeoffs. Proc. IEEE 98(12), 2185-2200 (2010) 